\title{
Prevalencia y perfil de sensibilización a aeroalérgenos en 1.199 niños asmáticos: serie consecutiva de casos
}

\author{
JAVIER MALLOL ${ }^{1}$, PABLO RABY ${ }^{1,2,3}$, DAVID CAMBIAZO $^{\text {a }}$, \\ CÉSAR PEÑALOZA ${ }^{\mathrm{a}}$, RICARDO PALMA ${ }^{\mathrm{a}}$, MANUEL DE ORÚE ${ }^{1}$

\section{Prevalence of atopy in 1,199 asthmatic children from southern Santiago, Chile}

Background: The prevalence of atopy in asthmatic children is widely variable around the world as demonstrated by large multicentric international studies. Aim: To determine the prevalence of atopy, defined as a positive reaction to one or more allergens in the skin prick test (SPT), in children with persistent asthma. Material and Methods: We studied 1,199 children (54\% male), aged between 4 and 16 years with confirmed diagnosis of asthma and followed at a Department of Pediatric Respiratory Medicine, between 2006 and 2011. SPT was performed according to international recommendations using standardized aeroallergens, in the forearm. A positive reaction was defined as a wheal $\geq 3 \mathrm{~mm}$ to one or more allergens. Results: The overall prevalence of atopy (positive SPT) was $49.4 \%$ (95\% confidence interval (CI) 46.5-52.2) and there was a significant trend towards a higher prevalence with increasing age $(p<0.01)$. The main allergens with positive reactions were Dermatophagoides with $24.9 \%$ (95\% CI 26.7-31.9), grass 24.0\% (95\% CI 21.6-26.5), weeds 19.0\% (95\% CI 16.9-21.4), cat $17.7 \%$ (95\% CI 15.4-20.2), and Alternaria with 11.0\% (95\% CI 9.1-13.1). Sixty five percent of positive children reacted to one or more allergens. There were no adverse reactions. Conclusions: In the southern metropolitan area of Santiago de Chile, half of children with asthma are sensitized to common aeroallergens.

(Rev Med Chile 2014; 142: 567-573)

Key words: Asthma; Child; Epidemiology; Hypersensitivity.

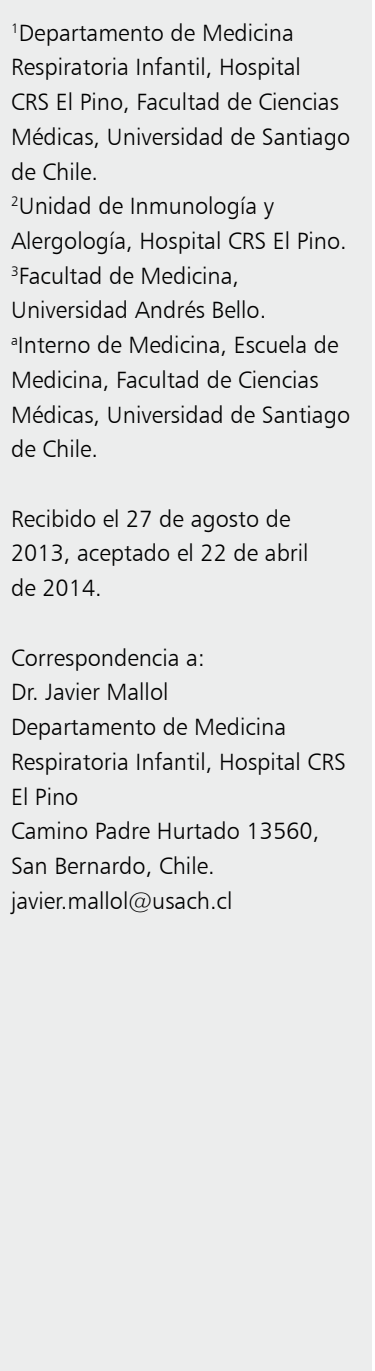

'Departamento de Medicina Respiratoria Infantil, Hospital CRS El Pino, Facultad de Ciencias Médicas, Universidad de Santiago de Chile.

¿Unidad de Inmunología y Alergología, Hospital CRS El Pino. ${ }^{3}$ Facultad de Medicina, Universidad Andrés Bello. anterno de Medicina, Escuela de Medicina, Facultad de Ciencias Médicas, Universidad de Santiago de Chile.

Recibido el 27 de agosto de 2013, aceptado el 22 de abril de 2014.

Correspondencia

Dr. Javier Mallo El Pino

Camino Padre Hurtado 13560

San Bernardo, Chile.

javier.mallol@usach.c
L a relación entre asma infantil y sensibilización atópica es conocida desde hace mucho $^{1,2}$, y aunque algunos estudios longitudinales han demostrado que la atopia está dentro del grupo de factores que predicen la persistencia del asma hacia la vida adulta ${ }^{3-6}$ y también su severidad $^{7}$, esos hallazgos no han sido consistentes ${ }^{8}$.

El asma es una condición altamente prevalente en la niñez ${ }^{9}$ pero a pesar de los significativos avances en varios aspectos de esta condición, los determinantes de su inicio, de sus características etiopatogénicas y de su evolución natural, aún permanecen pobremente comprendidos ${ }^{10}$. Aunque en la edad pediátrica el asma se asocia con frecuencia a atopia, algunos autores han reportado que su rol en el asma estaría sobreestimado ${ }^{11}$, habiéndose demostrado que la sensibilización a alérgenos comunes no se relaciona con la presencia de síntomas actuales de asma y que la fracción de asma atribuible a atopia varía enormemente entre distintas localidades ${ }^{12}$. Además, varios estudios diseñados para medir asma y atopia en la misma población en diferentes momentos, no han encontrado correlación entre los cambios de prevalencia de asma $\mathrm{y}$ atopia, de tal forma que aumentos significativos en el asma no se asociaron con aumentos en la prevalencia de atopia ${ }^{13,14} \mathrm{y}$ viceversa ${ }^{15}$.

En niños asmáticos, la prevalencia de sensibi- 
lización a alérgenos (evaluada con pruebas cutáneas) es también muy variable y de acuerdo con distintos estudios latinoamericanos oscila entre $25 \%$ y $63 \%^{16-18}$, sin embargo, los resultados de esos estudios no son comparables por diferencias en las poblaciones estudiadas y en su metodología. En 2 trabajos previos, también en la ciudad de Santiago, se ha evaluado la prevalencia de sensibilización en niños asmáticos, y en adolescentes con y sin sibilancias en los últimos 12 meses. En el primer trabajo, en un grupo de 237 niños asmáticos de 4 a 14 años de edad, se realizó prueba de punción cutánea (prick test o test cutáneo [TC]) con 39 alérgenos (aéreos y alimentarios) y se encontró una prevalencia de TC positivo de $62,5 \%$, sin diferenciar porcentajes de positividad a alérgenos alimentarios o aéreos ${ }^{16}$. En el otro trabajo, de tipo casos-controles, en muestras aleatorias de 100 adolescentes con y sin sibilancias en los últimos 12 meses desde una muestra a nivel de comunidad de 3.200 niños, la prevalencia de TC positivo a aeroalérgenos fue $44 \%$ en los con síntomas actuales de asma y $42 \%$ en los sin síntomas previos de asma ${ }^{17}$.

Debido a que las diferencias metodológicas impiden comparaciones y dificultan interpretaciones, y considerando el número relativamente bajo de niños en ambos estudios, creímos necesario actualizar la información en nuestra área en una población altamente seleccionada y en un mayor número de pacientes. Por lo tanto, este estudio se realizó para determinar la prevalencia y el patrón de sensibilización a aeroalérgenos (TC positivo) en una serie consecutiva de 1.199 niños asmáticos persistentes, derivados desde la atención primaria, en el área sur de Santiago de Chile (Departamento de Medicina Respiratoria Infantil, Hospital El Pino).

\section{Pacientes y Método}

Desde el año 2006 y hasta 2011, se realizó TC a todos los pacientes, mayores de 4 años de edad, derivados de atención primaria con diagnóstico de asma leve-moderada persistente ${ }^{19}$ al Departamento de Medicina Respiratoria Infantil del Hospital El Pino, en el área sur de Santiago de Chile. En total fueron 1.199 niños (4 a 16 años de edad). A todos ellos se les realizó prueba de punción cutánea o TC con aeroalérgenos de acuerdo a recomendaciones internacionales ${ }^{20}$.
El TC fue realizado en la cara anterior del antebrazo por un médico o enfermera entrenados en la técnica del examen, empleándose los siguientes alérgenos estandarizados: Alternaria, Aspergillus fumigatus, Penicillium notatum, cucaracha americana (Periplaneta americana), Dermatophagoides pteronyssinus, Dermatophagoides farinae, gato (epitelio) y perro (epitelio) (ALK-Abelló, España); árboles mezcla (arce, aromo, ciprés, plátano oriental), gramíneas mezcla (Festuca, Lolium, Cynodon) y malezas mezcla (Chenopodium, Plantago, Rumex, Taraxacum) (Nelco Labs., EE.UU.). Se usaron lancetas para TC con punta de $1 \mathrm{~mm}$ (ALK-Abelló, España); como control positivo se utilizó histamina $10 \mathrm{mg} / \mathrm{ml}$ (ALK-Abelló, España), y solución salina como control negativo. Fueron consideradas como respuestas positivas aquellas pápulas con un tamaño $\geq 3 \mathrm{~mm}$ sobre el control negativo.

\section{Análisis estadístico}

El análisis de los datos se realizó empleando estadística descriptiva mediante el software Stata v12.1. La prevalencia de sensibilización positiva (TC positivo) se calculó considerando el número de casos reactores positivos sobre el total de niños. La comparación de las proporciones de casos positivos por tipo de alérgeno se realizó mediante la prueba de $\chi^{2}$ de Pearson, en tanto que para comparar los porcentajes de TC positivo por año de edad, se empleó una prueba de comparaciones múltiples de Kruskal-Wallis. Los resultados se expresan como medias o porcentajes y sus correspondientes intervalos de confianza al 95\%; se consideró como estadísticamente significativo un valor de $\mathrm{p}<0,05$.

La asociación entre el porcentaje de TC positivo y las variables edad y sexo se evaluó empleando un modelo de regresión logística ajustado por edad y sexo. Para determinar los grupos de edad más diferenciados en cuanto a prevalencia de TC positivo (desde los 4 a los 16 años), se efectuó una técnica de suavizado mediante una regresión localmente ponderada o LOWESS (del inglés locally weighted scatterplot smoothing $)^{21}$.

\section{Resultados}

La edad promedio de los pacientes fue de 8,2 años (IC: 8,08-8,44), desde 4 a 16 años de edad, sin diferencias entre las proporciones de niños 


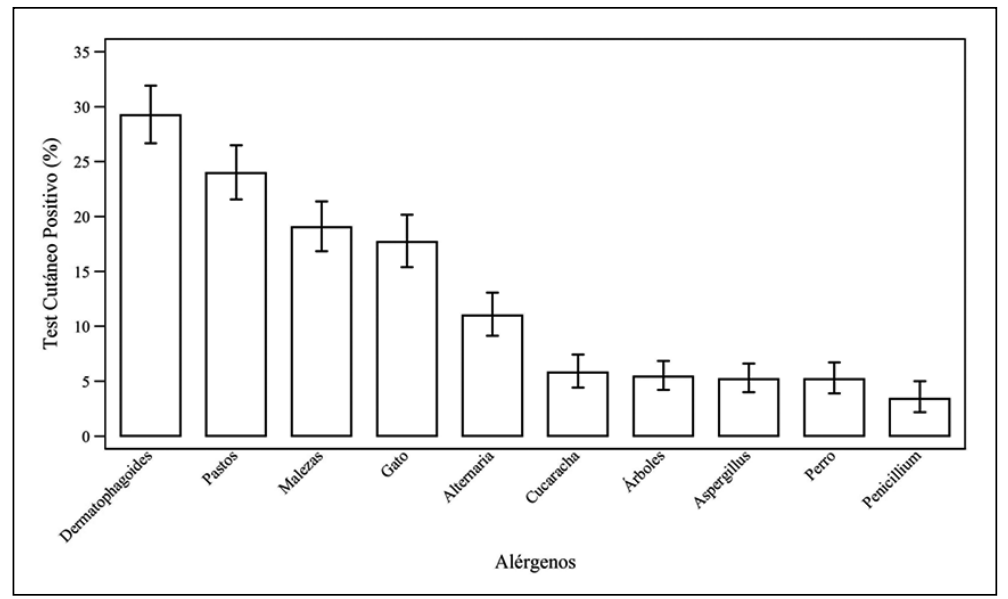

Figura 1. Perfil de sensibilización a aeroalérgenos evaluada mediante TC en niños con asma, ordenado por frecuencia (porcentaje promedio e IC95\%). TC: test cutáneo. $y$ niñas $(p=0,523)$; tampoco hubo diferencia significativa entre las proporciones de reactores positivos por sexo (masculino 51,3\%, femenino $47,8 \%),(\mathrm{p}=0,240)$.

Mil ciento ochenta y tres participantes $(98,7 \%)$ no presentaron reacción al suero fisiológico y de los 16 participantes que reaccionaron, 11 pacientes tuvieron reacción de $1 \mathrm{~mm}, 4$ de $2 \mathrm{~mm}$ y 1 de 3 $\mathrm{mm}$. Todos los participantes reaccionaron a la histamina, $0,33 \%$ presentó un diámetro de pápula menor a $3 \mathrm{~mm}$ y $2 \%$ presentó un diámetro igual o superior a $10 \mathrm{~mm}$.

El 49,4\% de los pacientes tuvo TC positivo y la sensibilidad a los alérgenos varió entre 3,4\% (Penicillium) y 29,2\% (Dermatophagoides). Las gramíneas $(24,0 \%)$ y las malezas $(19,0 \%)$, junto al epitelio de gato $(17,7 \%)$, fueron los alérgenos más frecuentemente positivos después de Dermatophagoides (Figura 1).

De los pacientes con TC positivo $(n=592)$, $36 \%$ fue reactivo sólo a uno de los alérgenos, mientras que $21 \%$ al menos lo fue a 4 alérgenos. Al evaluar la sensibilidad de los alérgenos en el grupo formado por los reactivos a un único alérgeno y en el grupo con hasta tres alérgenos reactivos, éstos presentaron la misma tendencia del grupo general, encabezados por Dermatophagoides y gramíneas.

La prevalencia de positividad a TC resultó diferente $(\mathrm{p}<0,001)$ entre los distintos grupos de edad (Figura 2), con una tendencia significativa a una mayor prevalencia con el incremento de la edad, yendo desde $32 \%$ hasta $61 \%$ en los niños de 4 a los de 16 años con un pico de $77 \%$ en los 15 años.

El resultado del suavizamiento para la pre- valencia de atopia sobre la edad determinó 4 pendientes (diferenciadas con las letras A, B, C y D) correspondientes a la formación de 4 grupos: 4 y 5 años (A), 6 y 7 años (B), 8 a 10 años (C) y de 11 a 16 años (D), los que presentan prevalencias diferentes $(\mathrm{p}<0,001)$ con valores de $31,9 \%$ $(26,3-37,4), 45,2 \%(39,6-50,8), 55,5 \%(49,8-61,1)$ y $62,7 \%(57,3-68,0)$ (Figura 3$)$.

Hubo una asociación significativa entre la sensibilización a alérgenos y la edad, con un riesgo significativamente mayor de sensibilización a medida que aumentó la edad de los niños $(\mathrm{OR}=1,15$; IC 95\% 1,11-1,19), $\mathrm{p}=0,000$; el sexo no se asoció con la prevalencia de TC positivo $(\mathrm{p}=0,250)$. La prevalencia para cada edad con su respectivo intervalo de confianza, según ajuste por el modelo,

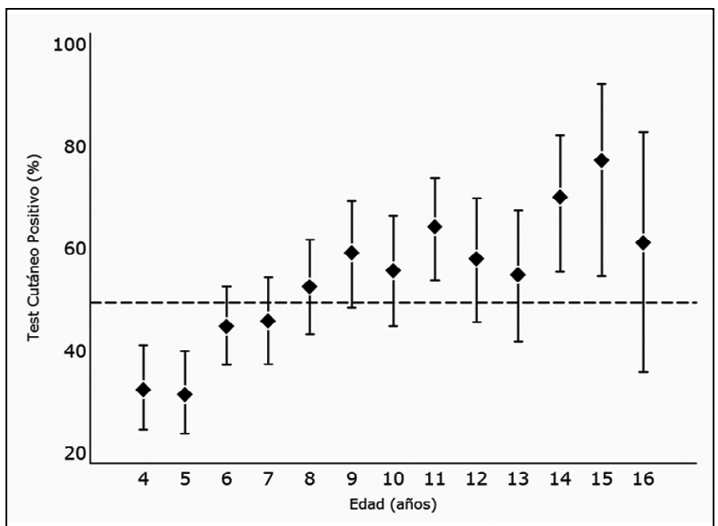

Figura 2. Prevalencia de TC positivo por edad. La línea semicontinua representa la prevalencia media general del TC positivo $(49,4 \%)$. TC: test cutáneo. 


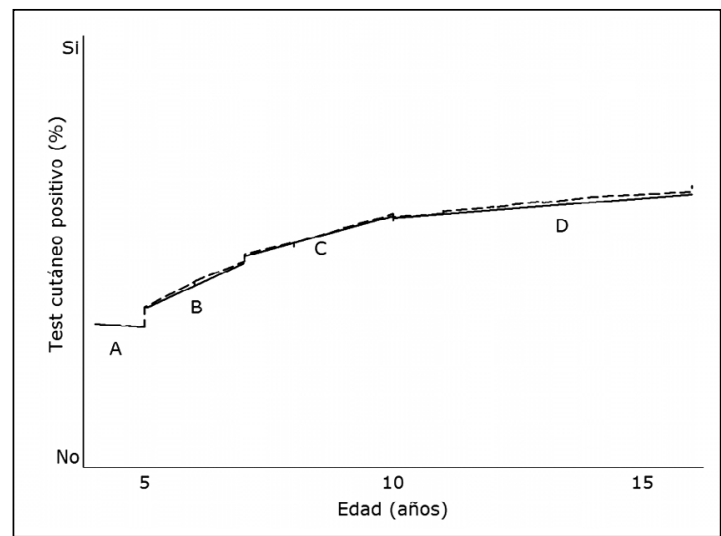

Figura 3. Agrupación de edades, según suavizamiento por regresiones ponderadas.

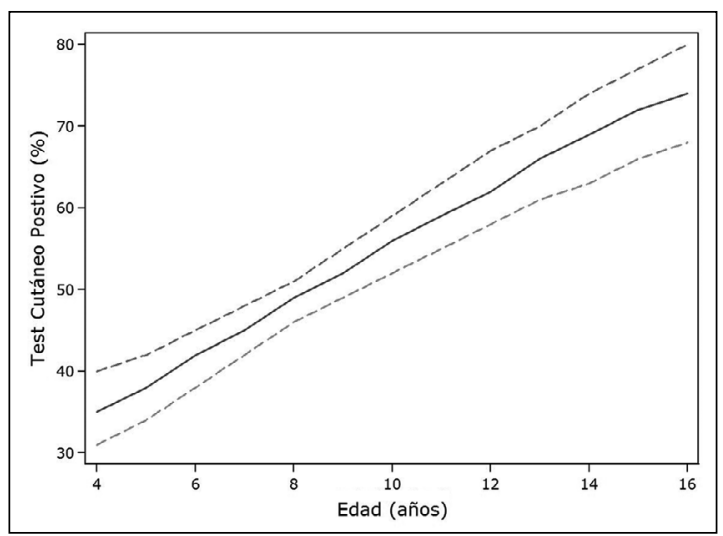

Figura 4. Estimaciones de la prevalencia de TC positivo ajustadas por un modelo de regresión logística según edad. TC: test cutáneo.

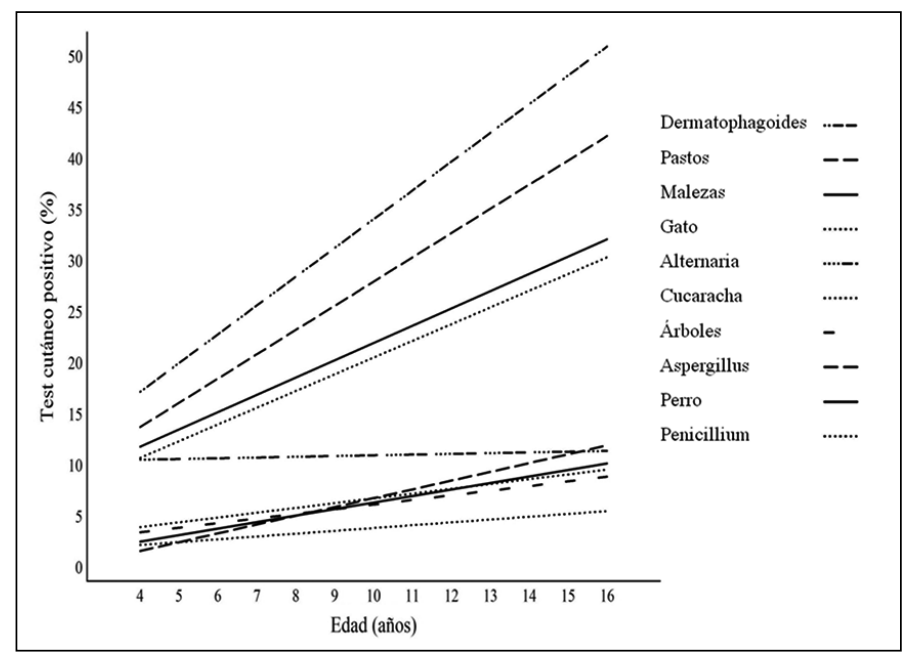

Figura 5. Prevalencia de TC positivo en niños asmáticos por grupo de edad y tipo de alérgeno. TC: test cutáneo. se presenta en la Figura 4. Las líneas de tendencia para la prevalencia de TC positivo en niños asmáticos por grupo de edad y tipo de alérgeno se observan en la Figura 5.

\section{Discusión}

Este trabajo encontró que $49,4 \%$ de los niños con asma persistente demostrada tuvo TC positivo a uno o más de los alérgenos empleados. Otro hallazgo fue la diferencia significativa de la prevalencia de sensibilización a los aeroalérgenos en los distintos grupos de edad, observándose una mayor prevalencia en los grupos etáreos mayores, especialmente para Dermatophagoides, malezas, gramíneas y gato. En nuestro estudio los alérgenos más frecuentemente positivos fueron Dermatophagoides, gramíneas, malezas y gato; encontrándose que la mayoría $(35,5 \%)$ de los pacientes que reaccionaron positivamente lo hicieron a un solo alérgeno.

Estas cifras son similares a lo descrito previamente por nosotros en esta misma área de Santiago $^{17}$; sin embargo, es más baja que la comunicada por otros autores en esta misma ciudad ${ }^{16}$, quienes 
en un estudio de 237 niños asmáticos encontraron que $62,5 \%$ tenían asma atópica. Leung en Hong Kong, en un estudio de 204 niños asmáticos de una edad promedio similar a la de nuestro grupo $(8,2$ años), encontró una prevalencia de TC positivo de $83 \%$, predominantemente a ácaros del polvo de habitación ${ }^{22}$. García-Marcos et al en un estudio realizado en tres ciudades españolas, en niños de 9-12 años, encontraron que $62,4 \%$ de los niños con síntomas actuales de asma fueron atópicos ${ }^{23}$. En Pretoria ${ }^{24}, 45 \%$ de los asmáticos tuvieron TC positivo, y el alérgeno más comúnmente positivo fue el pasto Bermuda (22\%) seguido por cucaracha $(11 \%)$, perro $(10 \%)$ y ácaros del polvo de habitación $(8 \%)$.

La amplia variabilidad de la prevalencia de sensibilización a alérgenos en niños, tanto en poblaciones seleccionadas como a nivel general ${ }^{11,12}$, indica que el ambiente es crucial para determinar la prevalencia y el perfil de sensibilización alérgica en poblaciones humanas. Eso ha sido demostrado por Ronchetti et $\mathrm{al}^{25}$, quienes encontraron una alta correlación entre la atopia en niños asmáticos y no asmáticos lo cual sugiere fuertemente que la prevalencia de atopia en el asma depende de factores ambientales que inducen simultáneamente atopia en pacientes asmáticos y en los sujetos no asmáticos.

El más grande de los estudios internacionales de asma y alergias en la niñez (ISAAC), en su segunda fase, estudió las relaciones entre atopia y síntomas actuales de asma (30 centros en 22 países, TC en 31.759 escolares de 8-12 años), y no encontró correlación entre sensibilización alérgica y síntomas de asma ${ }^{12}$. La fracción de sibilancias en los últimos 12 meses atribuible a sensibilización atópica varió desde $0 \%$ en Ankara (Turquía) a 93,8\% en Guangzhou (China); también se encontró que en los países desarrollados $41 \%$ de los síntomas actuales de asma son atribuibles a la atopia. En un estudio realizado en escolares chilenos sanos y con síntomas actuales de asma, nosotros demostramos que la reactividad bronquial en niños con y sin síntomas actuales de asma puede estar aumentada sin que haya un aumento concomitante en la atopia ${ }^{17}$.

Desde el punto de vista clínico, es difícil diferenciar con certeza entre niños asmáticos atópicos y no atópicos. Algunos autores a nivel local (Santiago) no encontraron diferencias significativas en la severidad de las exacerbaciones ni en la función pulmonar, entre niños asmáticos atópicos y no atópicos; sólo hubo un mayor número de tratamientos con corticoides orales asociado al asma atópica ${ }^{16}$. Asimismo, en un estudio reciente realizado en 321 niños asmáticos en Estados Unidos de Norteamérica, en el cual 2 tercios de los pacientes tenían TC positivo a uno o más alérgenos, se encontró que la historia familiar de alergia, edad de comienzo del asma, exposición a tabaco, sexo, obesidad o severidad del asma, no permitían distinguir a los niños con asma alérgica y no alérgica ${ }^{26}$. Por otra parte, se ha demostrado que los cambios patológicos típicos del asma están igualmente presentes en niños asmáticos atópicos y no atópicos ${ }^{27}$.

En nuestro estudio se observó un significativo aumento de la sensibilización a alérgenos a medida que aumentaba la edad, lo cual también ha sido reportado en niños alérgicos con distintas condiciones (rinitis, eczema) ${ }^{28,29}$. La misma observación ha sido hecha en niños de población general seguidos longitudinalmente hasta los 18 años demostrándose un aumento significativo de la sensibilización alérgica evaluada por TC entre los 4 y 18 años (19,6\% a los 4 años, 26,9\% a los 10 años y $41,4 \%$ a los 18 años) $)^{30}$. En esa cohorte se encontró que la mayoría de los niños que no estaban sensibilizados a los 4 años permanecían así hasta la adolescencia y que la presencia de sensibilización alérgica a los 4 años era predictiva de sensibilización a otros alérgenos más tarde en la niñez ${ }^{31}$.

En el asma de la niñez esta observación podría explicarse clásicamente por una eventual relación directa entre el tiempo de exposición a alérgenos y sensibilización. Sin embargo, algunos autores ${ }^{32}$ han puesto en duda el modelo unidireccional tradicional para interpretar la asociación entre atopia y asma, y han propuesto que la asociación entre atopia y asma concordaría, al menos en parte, con un modelo alternativo en el que el desarrollo de las anomalías epiteliales asociadas al asma, predispone a las vías aéreas a una sensibilización alérgica local que tendría el efecto de agravar la respuesta inflamatoria y de remodelado.

En los últimos años, las infecciones respiratorias virales, en especial por rinovirus y en los primeros años de la vida, han recibido atención creciente como causa o coadyuvante de la activación del epitelio de las vías aéreas y la potenciación asociada de la síntesis local de mediadores que 
Prevalencia y sensibilización a aeroalérgenos en niños asmáticos - J. Mallol et al

resultarían en la polarización de las células Th2 y la posterior producción de $\operatorname{IgE}^{33}$. Se ha demostrado que la IL-25 producida por las células epiteliales juega un rol clave en la inducción de una respuesta inflamatoria tipo Th2 después de las infecciones virales $^{34}$, aunque se ha encontrado que la sensibilización alérgica se asocia positivamente con el rinovirus y no con otros virus ${ }^{35}$. Recientemente, Baraldo et $\mathrm{al}^{36}$ en un estudio inmunohistoquímico de biopsias bronquiales de niños, demostraron que los asmáticos (atópicos y no atópicos) y los atópicos (sin asma) tienen como factor común una baja producción de interferón gamma frente a infecciones por rinovirus.

Las limitaciones de nuestro estudio son aquellas comunes a los estudios transversales; además, como en todos los trabajos que incluyen poblaciones seleccionadas, los hallazgos no debieran generalizarse $a$ priori. Es posible que niños asmáticos de similares características de severidad que los de este estudio, pero viviendo en otras comunas de la Región Metropolitana, presenten prevalencia y perfil de sensibilización diferentes. Aun así, los resultados del presente estudio realizado en una serie consecutiva de 1.199 niños con asma persistente, aportan datos útiles para aumentar el conocimiento local con respecto a la prevalencia de sensibilización alérgica en estos pacientes.

La proporción de niños asmáticos que tiene atopia asociada, en nuestro medio, es posible que varíe de acuerdo a distintas circunstancias socioeconómicas y ambientales. No obstante, es muy relevante determinar la presencia de atopia en estos pacientes asmáticos puesto que estudios longitudinales ${ }^{3-7}$ han mostrado que ésta constituye un importante factor de riesgo para la continuación del asma hacia la adultez y para la recaída en pacientes que están en remisión.

\section{Referencias}

1. von Mutius E. Environmental factors influencing the development and progression of pediatric asthma. J Allergy Clin Immunol 2002; 109 (6 Suppl): S525-32.

2. Custovic A, Simpson A. Environmental allergen exposure, sensitisation and asthma: from whole populations to individuals at risk. Thorax 2004; 59 (10): 825-7.

3. Strachan DP, Butland BK, Anderson HR. Incidence and prognosis of asthma and wheezing illness from early childhood to age 33 in a national British cohort. BMJ 1996; 312 (7040): 1195-9.
4. Godden DJ, Ross S, Abdalla M, McMurray D, Douglas A, Oldman D, et al. Outcome of wheeze in childhood. Symptoms and pulmonary function 25 years later. Am J Respir Crit Care Med 1994; 149 (1): 106-12.

5. Kokkonen J, Linna O. The state of childhood asthma in young adulthood. Eur Respir J 1993; 6 (5): 657-61.

6. Sears MR, Greene JM, Willan AR, Wiecek EM, Taylor DR, Flannery EM, et al. A longitudinal, populationbased, cohort study of childhood asthma followed to adulthood. N Engl J Med 2003; 349 (15): 1414-22.

7. Wolfe R, Carlin JB, Oswald H, Olinsky A, Phelan PD, Robertson CF. Association between allergy and asthma from childhood to middle adulthood in an Australian cohort study. Am J Respir Crit Care Med 2000; 162 (6): 2177-81.

8. Gerritsen J, Koëter GH, Postma DS, Schouten JP, Knol K. Prognosis of asthma from childhood to adulthood. Am Rev Respir Dis 1989; 140 (5): 1325-30.

9. The ISAAC Steering Committee. Worldwide variations in the prevalence of asthma symptoms: the International Study of Asthma and Allergies in Childhood (ISAAC). Eur Respir J 1998;12 (2): 315-35.

10. Bush A. How early do airway inflammation and remodeling occur? Allergol Int 2008; 57 (1): 11-9.

11. Pearce N, Pekkanen J, Beasley R. How much asthma is really attributable to atopy? Thorax 1999; 54 (3): 268-72.

12. Weinmayr G, Weiland SK, Björkstén B, Brunekreef B, Büchele G, Cookson WO, et al; ISAAC Phase Two Study Group. Atopic sensitization and the international variation of asthma symptom prevalence in children. Am J Respir Crit Care Med 2007; 176 (6): 565-74

13. Peat JK, Haby M, Spijker J, Berry G, Woolcock AJ. Prevalence of asthma in adults in Busselton, Western Australia. BMJ 1992; 305 (6865): 1326-9.

14. Peat JK, van den Berg RH, Green WF, Mellis CM, Leeder SR, Woolcock AJ. Changing prevalence of asthma in Australian children. BMJ 1994; 308 (6944): 1591-6.

15. von Mutius E, Weiland SK, Fritzsch C, Duhme H, Keil U. Increasing prevalence of hay fever and atopy among children in Leipzig, East Germany. Lancet 1998; 351 (9106): 862-6.

16. Castro-Rodríguez JA, Ramírez AM, Toche P, Pavon D, Pérez MA, Girardi G, et al. Clinical, functional, and epidemiological differences between atopic and nonatopic asthmatic children from a tertiary care hospital in a developing country. Ann Allergy Asthma Immunol 2007; 98 (3): 239-44.

17. Mallol J, Castro-Rodríguez JA, Cortez E, Aguirre V, Aguilar P, Barrueto L. Heightened bronchial hyperresponsiveness in the absence of heightened atopy in children with current wheezing and low income status. 
Thorax 2008; 63 (2): 167-71.

18. Souza da Cunha S, Barreto ML, Fiaccone RL, Cooper PJ, Alcantara-Neves NM, Simões SM, et al. Asthma cases in childhood attributed to atopy in tropical area in Brazil. Rev Panam Salud Publica 2010; 28 (6): 405-11.

19. National Asthma Education and Prevention Program. Expert Panel Report 3 (EPR-3): Guidelines for the Diagnosis and Management of Asthma-Summary Report 2007. J Allergy Clin Immunol 2007; 120 (5 Suppl): S94-138.

20. Sub-Committee on Skin Tests of the European Academy of Allergology and Clinical Immunology. Position paper: Allergen standardization and skin tests. Allergy 1993; 48 (14 Suppl): 48-82.

21. Cleveland WS. Robust locally weighted regression and smoothing scatterplots. J Am Stat Assoc 1979; 74 (368): 829-36.

22. Leung TF, Li AM, Ha G. Allergen sensitisation in asthmatic children: consecutive case series. Hong Kong Med J 2000; 6 (4): 355-60.

23. García-Marcos L, Castro-Rodríguez JA, Suárez-Varela MM, Garrido JB, Hernández GG, Gimeno AM, et al. A different pattern of risk factors for atopic and nonatopic wheezing in 9-12-year-old children. Pediatr Allergy Immunol 2005; 16 (6): 471-7.

24. Els C, Boonzaaier L, Green RJ. Atopy in asthmatic children attending a tertiary hospital in Pretoria. Curr Allergy Clin Immunol 2010; 23: 180-2.

25. Ronchetti R, Rennerova Z, Barreto M, Villa MP. The prevalence of atopy in asthmatic children correlates strictly with the prevalence of atopy among nonasthmatic children. Int Arch Allergy Immunol 2007; 142 (1): 79-85.

26. Sinisgalli S, Collins MS, Schramm CM. Clinical features cannot distinguish allergic from non-allergic asthma in children. J Asthma 2012; 49 (1): 51-6.

27. Turato G, Barbato A, Baraldo S, Zanin ME, Bazzan E, Lokar-Oliani K, et al. Nonatopic children with multi- trigger wheezing have airway pathology comparable to atopic asthma. Am J Respir Crit Care Med 2008; 178 (5): 476-82.

28. Garde J, Hervás D, Marco N, Milan JM, Dolores Martos M. Calculating the prevalence of atopy in children. Allergol Immunopathol (Madr) 2009; 37 (3): 129-34.

29. Martínez J, Méndez C, Talesnik E, Campos E, Viviani P, Sánchez I. [Skin prick test of immediate hypersensitivity in a selected Chilean pediatric population sample]. Rev Med Chile 2005; 133 (2): 195-201.

30. Ziyab AH, Karmaus W, Yousefi M, Ewart S, Schauberger E, Holloway JW, et al. Interplay of filaggrin loss-offunction variants, allergic sensitization, and eczema in a longitudinal study covering infancy to 18 years of age. PLoS One 2012; 7 (3): e32721.

31. Roberts G, Zhang H, Karmaus W, Raza A, Scott M, Matthews S, et al. Trends in cutaneous sensitization in the first 18 years of life: results from the 1989 Isle of Wight birth cohort study. Clin Exp Allergy 2012; 42 (10): 1501-9.

32. Guerra S, Martínez FD. Is allergy an asthmatic disease? Arch Bronconeumol 2011; 47 (10): 479-81.

33. Holgate ST, Arshad HS, Roberts GC, Howarth PH, Thurner P, Davies DE. A new look at the pathogenesis of asthma. Clin Sci (Lond) 2009; 118 (7): 439-50.

34. Kaiko GE, Phipps S, Angkasekwinai P, Dong C, Foster PS. NK cell deficiency predisposes to viral-induced Th2type allergic inflammation via epithelial-derived IL-25. J Immunol 2010; 185 (8): 4681-90.

35. Jartti T, Kuusipalo H, Vuorinen T, Söderlund-Venermo $\mathrm{M}$, Allander T, Waris $\mathrm{M}$, et al. Allergic sensitization is associated with rhinovirus-, but not other virus-, induced wheezing in children. Pediatr Allergy Immunol 2010; 21 (7): 1008-14.

36. Baraldo S, Contoli M, Bazzan E, Turato G, Padovani A, Marku B, et al. Deficient antiviral immune responses in childhood: Distinct roles of atopy and asthma. J Allergy Clin Immunol 2012; 130 (6): 1307-14. 